\title{
REVIEW
}

\section{Use of human patient simulation to teach emergency medicine trainees advanced airway skills}

\author{
Craig Ellis, Geoff Hughes
}

Advanced airway management skills are essential for emergency physicians and are a required competency for trainees sitting for the Fellowship of the Australasian College for Emergency Medicine. The technical psychomotor skills are best taught under supervision in the operating theatre or the emergency department, but the decision making and organisational skills needed to apply them safely and manage their complications are harder to teach.

The use of human patient simulators, which model human physiology and the effects of aging, illness and drugs, is now a common method of teaching crisis management to anaesthetic trainees. It is our view that this is an ideal environment to teach the integration of the basic technical skills of airway management, with the goal of trying to manage airway problems in the emergency department.

This paper describes a one day advanced airway course we developed and ran, primarily for emergency medicine trainees. It included lectures and skill stations centred on a human patient simulator.

\section{Patient simulation}

Patient simulators have evolved over the last 20 years from simple manikins for teaching cardiopulmonary resuscitation to more complex advanced cardiac life support (ACLS) manikins, which can be defibrillated and intubated. A recent evolutionary step from these ACLS simulators has been the development of physiological modelling simulators. These are human shaped computerised manikins that can be used for complex advanced life support skills training. Importantly, they simulate normal human physiology. They breath with chest wall movement, have a pulse, breath and heart sounds, produce urine, and can be monitored for variables such as electrocardiography, end tidal carbon dioxide, oxygen saturation, heart rate, and blood pressure. The physiological model can be adjusted to reflect disease states, respond appropriately to administered drugs, and alter physical findings, as well as monitored parameters.

The principle of realistic simulation of emergency situations has evolved from the training of airline pilots. For the last 25 years flight simulation has been a required standard for teaching commercial pilots, and all active pilots are required to attend refresher simulator assessments. By repeatedly being exposed to accurate simulation of real situations and emergencies, it enables the pilot to respond appropriately when the real situation arises. The same is true of human simulation. During simulation steps are taken to ensure that things are as realistic as possible-ambulance handover, similar physical environment and noise, hysterical relatives, equipment failures or losses, losing staff to other emergencies, etc. Anecdotally it seems the greater the fidelity of the simulator, the more the participants are "lost" within the simulation.

Thus they allow trainees to experience and manage crises (some extremely rare) in an environment where no patient is at risk and mistakes can be reviewed. The simulation can also be video recorded and later used in a debriefing session. Video review of a performance can be a personally uncomfortable experience, but it is rated as a positive experience by participants in crisis management and airway courses. ${ }^{12}$

Patient simulators have been embraced by the anaesthetic community. ${ }^{2-4} \mathrm{~A}$ group from Stanford University did much of the original work on the development of anaesthetic simulators and their application to anaesthetic education. They developed the original anaesthetic crisis resource management course, ${ }^{2}$ which evolved from the cockpit resource management course developed by the airline industry. It uses simulated anaesthetic crises, combined with instruction in crisis management and a comprehensive debriefing of the video taped simulations, to teach crisis management skills to anaesthetists.

In the Australasian centres that have simulators (Wellington, Sydney, Melbourne, Perth), they are used extensively for teaching crisis management to anaesthetic trainees and for continuing medical education for consultants. There is little published about their use outside of anaesthesia, although their use in teaching emergency medicine is increasing.

The Wellington simulator, a METI-HPS (figs 1 and 2), is one of two commercially available models of human patient simulators, the 


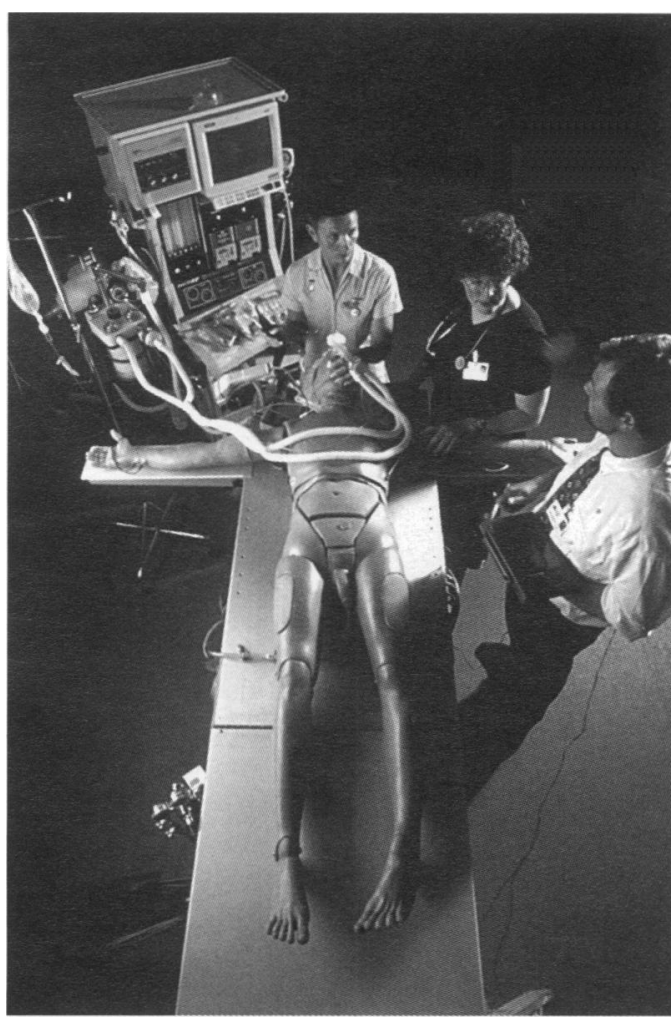

Figure 1 Wellington simulator: view from above. Trainees with an instructor.

other being produced by Eagle Technology. It utilises a modified Windows platform, programmed in Turbo-C. It runs on a script controlled, model driven basis.

Script controlled simulation has previously been used in most computer simulations, a process where the computer gives a certain response, in reaction to a certain event. To have maximal realism the script needs to be complex, and to allow for other variables. For example it is insufficient to plan for the cardiovascular responses to a certain dose of an induction agent. The program must allow for possible interactions with other systems or other drugs. This complexity limits the use of scripting; it is simply not possible to script for every permutation of variables.

To overcome these limitations, the METIHPS uses a combination of scripting and mathematical physiological modelling. The computer runs complex models of the main physiological systems-for example, cardiovascular, respiratory, renal. It integrates the different system models and uses the knowledge of how pathological states and administered drugs alter normal physiology to produce a response. Overall scenario control can be script driven or modified by an operator. This allows for a more dynamic response, allowing for hundreds of variables to be included in the overall simulator response. ${ }^{5}$

\section{Airways management}

In the early management of the critically injured and ill patient the primary concern is airway security. The gold standard is endotracheal intubation, using rapid sequence induction (RSI): the use of sedative agents and neuromuscular blocking agents to facilitate intubation. Traditionally this has been done by anaesthetists. However with the evolution of emergency medicine specialists and extended consultant cover in the emergency department, it is now an obligatory skill for emergency physicians. In the United States the majority of emergency department intubations are performed by emergency physicians. ${ }^{6-9}$ Recent studies demonstrated that emergency department intubation by emergency medicine physicians, is safe with low complication rates. The final report of the National Emergency Airway Registry pilot project found $93 \%$ of adult (and $57 \%$ paediatric, $79 \%$ if including paediatric emergency physicians) intubations were performed by emergency physicians, with a serious complication rate of $3 \%$. It also demonstrated that RSI was superior to sedation alone, intubation without any sedation, or nasopharyngeal intubation. ${ }^{8} \mathrm{~A}$ study by University of California Davis Medical Center of 610 emergency department intubations was similar, with $97 \%$ of intubations performed by emergency physicians. They had a success rate of $98.9 \%$ and a complication rate of $9.3 \%$, most of which were minor (such as transient desaturation or hypotension and dental injuries). The surgical airway rate was $1.1 \% .^{9}$ These figures compare favourably with anaesthetists performing intubations in critically ill patients. ${ }^{10}$ Another study of 98 emergency residency programmes, found only $7 \%$ mandated anaesthetic involvement during intubations, with $41 \%$ not involving anaesthetists in emergency department intubations. ${ }^{6}$

The technical skills of intubation can be learnt by experience in the operating room, but the protocol of emergency department RSI and the management of complications are more difficult to learn. There is often a reluctance to allow junior trainees to perform the procedure and when things go wrong the most experienced person is obliged to take over.

We believe that realistic patient simulation provides an opportunity to develop the necessary crisis management and organisational skills to undertake safe emergency department RSI intubations. We designed a one day course for emergency trainees. We believe this is the first of its type in Australasia or the UK.

\section{Advanced airways course}

The course aim was to teach the theoretical and organisational skills of RSI and the management options for critical problems. It also reviewed the basic technical skills of intubation and the use of airway adjuncts.

There were 12 participants, working in groups of six. The participants comprised seven emergency medicine trainees, one surgical trainee, and two junior anaesthetic senior house officers. There were also two experienced paramedics, included to extend the perspective to pre-hospital airway management issues and to assess the value of the patient simulator in teaching pre-hospital emergency medicine. The faculty consisted of emergency medicine and anaesthetic consultants, includ- 


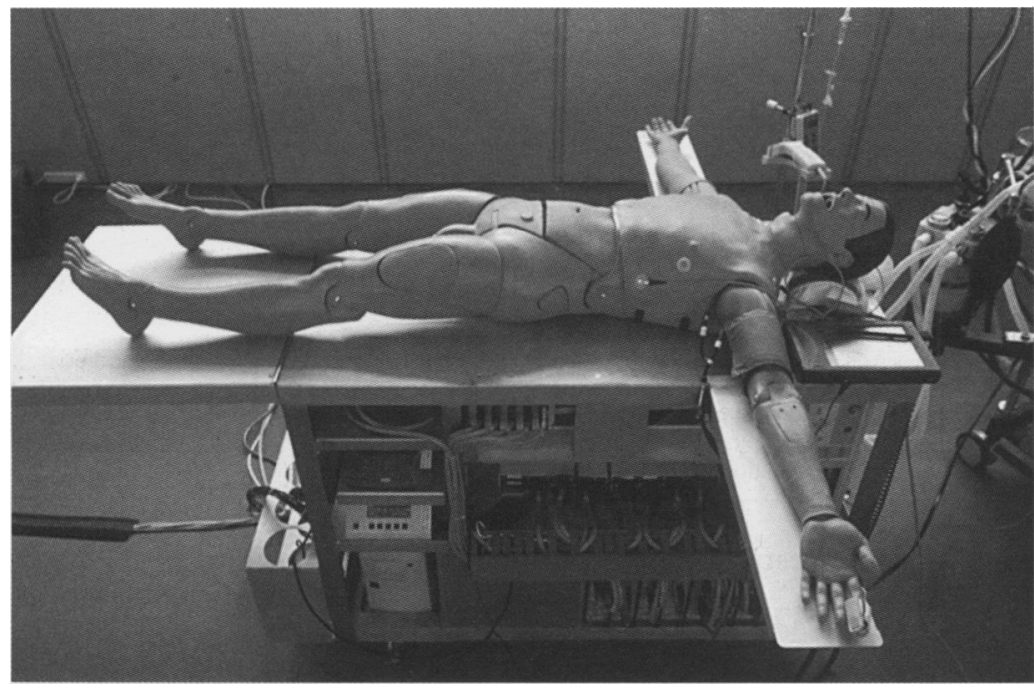

Figure 2 Wellington simulator: side view to show equipment under manikin.

ing an Australian emergency physician certified as an anaesthetic crisis resource management instructor.

The 10.5 hour programme covered the theory of RSI and its pharmacology, assessment of a difficult airway, management of a failed intubation (including surgical airways), and the paediatric airway. There was an initial introductory session to the use of the simulator, which was followed by scenario sessions. All participants were sent detailed pre-course reading.

We developed four scenarios. They were designed to represent common emergency department presentations needing advanced airway skills. The physiological responses for each scenario were programmed into the simulator directly or adapted from existing anaesthetic modules. The candidates were given an "ambulance officer handover" on each patient, and had to gain further information by assessing the simulated patient and starting appropriate monitoring. All the standard emergency department physiological variables (electrocardiography, oxygen saturation, non-invasive blood pressure, end tidal carbon dioxide) could be monitored.

All participants completed scenario 1 , as team leader, which let each control an uneventful RSI. In the remaining scenarios roles were rotated, enabling the participants to each undertake the role of team leader in one of the scenarios, and to be involved in the remainder as team members. In scenarios 2-3, the simulation controller had some freedom to modify the situation as scenarios evolved. These included a fall in blood pressure at induction, failed intubation, bronchial intubation, an undetected pneumothorax, and a cardiac arrest. The person in the team leader role was responsible for the decision and timing of intubation, the choice of drugs, airway vigilance, and coordination of the intubation.

- Scenario 1: 25 year old male involved in a motorcycle accident; isolated head injury; Glasgow coma score 9/15; uncooperative and aggressive.

- Scenario 2: 68 year old female involved in a high speed motor vehicle accident; multiple injuries; haemodynamically unstable; compromised airway.

- Scenario 3: 18 year old female after a tricyclic overdose who arrives in rapid pulse producing ventricular tachycardia; deteriorates to pulseless ventricular tachycardia.

- Scenario 4: 35 year old male in severe respiratory distress; uncertain cause; found to be upper airway foreign body.

\section{Analysis of the course}

The response to this course was very positivethe commonest request was for more simulator time. The main lesson we learned was that the decision pathways for the complicated scenarios need to be planned in advance, so the controller can respond in a more defined way. During a simulation events can unfold extremely rapidly as decisions are made and certain actions taken. We found that our scripting of the scenarios was slightly too superficial to allow the controller to be consistent across all candidates in any given situation. This problem was minimised due to the underlying modelling and responses generated by the simulator that were consistent across all participants.

On this initial pilot course there was no formal assessment of the candidates performance or comparison between them, although a critical event and error summary, adapted from one of the anaesthetic simulation modules, was used. Its role was to identify discussion points for the scenario debriefing rather than as a specific rating tool. The difficulty of assessing performance on a simulator is addressed in the discussion.

The commonest clinical errors identified by observation during the scenarios were:

- Prolonged periods of poor oxygenation before intubation or airway control.

- Failure to prepare drugs before the induction.

- Forgetting ACLS algorithms and advanced trauma life support principles.

- Failure to maintain cricoid pressure during induction.

These errors were made by all of the participants to greater or lesser degrees. There did not appear to be a difference between specialties or between scenarios.

Less common errors included:

- Failure to consider drug reactions/ anaphylaxis as a cause for rapid deterioration after intubation.

- Administration of neuromuscular blocking agents before team members were ready to proceed.

- Release of in-line immobilisation during induction.

- Role confusion with the identified team leader/"intubator" for each intubation; others tried to lead. This led to conflict about drugs and timing of their administration.

- Poor airway vigilance after intubation. Attention was easily distracted from ensuring the tube and circuit remained secured.

Overall the frequency of errors was fairly constant over all four scenarios. The final scenario involving the upper airway foreign body was probably the least well performed, with 
Table 1 Bloom's cognitive educational objectives" (with examples relating to RSI)

1. Knowing: Knows basic facts and definitions

For example, correct dose of suxamethonium

2. Comprehending: Understands significance of basic facts and can interpret written and graphical data, and summarise/paraphrase that information

For example, indications and contraindications to use of suxamethonium

3. Applying: Applies information to new situations, can manipulate the information, and predict consequences For example, able to use suxamethonium in a real or simulated situation

4. Analysing: Breaks down information into its constituent parts, recognises relationships, and checks for consistency of data against a hypothesis

For example, establishes a cause for hypotension after administration of suxamethonium

5. Synthesising: Draws information together from multiple sources and integrates them, and produces a plan or a hypothesis For example, responds appropriately to hypotension after suxamethonium

6. Evaluating: Ability to make judgments about the value of ideas, based on internal consistency, external reference, or logical accuracy

For example, reflects upon and makes a logical evaluation of the treatment reponse

consistently the longest length of time to establishment of a definitive airway.

From these observations we identified four lessons.

- The importance of application of the of advanced life support principles to all emergency situations (even simulated ones).

- The importance of team work with a clearly identified team leader.

- The importance of familiarity with the pharmacokinetics and dynamics of drugs.

- The importance of technique in RSI and airway vigilance.

These are all basic and well established concepts. It was a sobering experience for several participants to realise how easily mistakes occur.

The choice of induction and neuromuscular blocking agents were generally appropriate for the scenarios presented. Some constantly displayed the reflex thinking of "thiopentone [thiopental] and suxamethonium", regardless of the clinical situation, and despite the pharmacology lecture and pre-course reading material. Most participants identified the organisational and procedural points required for a safe RSI.

\section{Discussion}

Bloom's cognitive theory of learning identifies six levels of educational objectives (table 1). ${ }^{11}$ These levels of learning apply well to clinical medical education and the application of this cognitive model can be applied to help define a role for simulation in the teaching of aspects of emergency medicine.

In our opinion human patient simulation has two discrete roles in teaching emergency medicine trainees within this cognitive model. At lower levels, it can instruct trainees in specific skills, and demonstrate physiological responses to drugs and illness more dynamically than a textbook description. At higher levels, it provides the opportunity to learn individual skill integration in dealing with complex problems, within a team environment.

It also has a role in the continuing medical education for emergency medicine specialists in crisis management.

In addition to its teaching role, the simulator helps in the formal assessment of skills and knowledge. Multiple choice questions and essay questions assess only factual knowledge, without the integration of these facts. Methods such as the objectively structured clinical examinations go some way towards assessing more complete integration. These are still limited by a lack of reality. A patient simulator can address this. In addition to knowledge, its assessment of the integration, application, and evaluation of that knowledge (Bloom's levels 3-6), goes beyond any other current assessment procedure. It allows candidates to demonstrate how they integrate knowledge in complex problems and how they evaluate and incorporate into their everyday practice.

There is currently debate in the anaesthetic literature over what is the best way to assess crisis management skills using simulators. Assessment of simple technical skills is straightforward. The assessment of performance in a crisis management simulation remains subjective. There are different ways to achieve the same end. The combination of an assessment, which assesses both crisis behaviours and technical skills, may provide the most reliable objective measure. The main problem has been variability between examiners. Devitt et al have proposed a system which appears to be consistent across assessors and to provide experience level discrimination. ${ }^{12}{ }^{13}$

\section{Conclusion}

It is our opinion that the use of patient simulation is the ideal way to integrate individual skills into the context of managing an emergency situation in the emergency department. It provides a dynamic and interesting learning environment, which at the same time is safe for patients and trainees. In the case of RSI it lets the trainee integrate the practical skills of intubation in the environment of an emergency department airway problem.

The technology of patient simulators continues to evolve rapidly and as their degree of fidelity increases so does their value as a teaching tool. Work that has already been done in using simulation to teach crisis management in anaesthesia can act as a starting point for developing its further use in emergency medicine.

We plan to continue running this course, as well as developing additional courses for crisis management in emergency medicine.

We would like to thank Dr Brian Robinson, Director of the National Patient Simulator, Wellington Hospital, Wellington, New Zealand and Dr Stephen Priestly, Emergency Medicine Programme Director, Southern Health Care Network Simulation Centre, Melbourne, Australia.

Conflict of interest: none.

Funding: we would like to acknowledge the generous support of Hoechst Marion Roussel, who provided sponsorship for the workshop. 
1 Kardash K, Tessler MJ. Videotape feedback in teaching laryngoscopy. Can 7 Anaesth 1997;44:54-8.

2 Howard S.K, Gaba DM, Fish KJ. Anaesthetic crisis resource management: teaching anaesthesiologists to handle critical incidents. Aviat Space Environ Med 1992;63:763.

3 Riley RH, Wilkes DH, Freeman JA. Anaesthetist's attitudes to an anaesthesia simulator. A comparative survey: USA and Australia. Anaesth Intensive Care 1997;25:514-19.

4 Kurrek MM, Fish KJ. Anaesthesia crisis resourse management training: an intimidating concept, a rewarding experience. Can $\mathcal{f}$ Anaesth 1996;43:430-4.

5 Van Meurs WL, Good ML, Lampotang S. Functional anatomy of full-scale patient simulators. $\mathcal{F}$ Clin Monit 1997;13: omy of

$6 \mathrm{Ma} \mathrm{O}$, Bentley B, Debehnke D. Airway management practices in emergency medicine residencies. Am $\mathcal{F}$ Emerg Med 1995;13:501-4.

7 Gallagher EJ, Coffey J, Lombardardi G, et al. Emergency procedures important to the training of emergency medicine residents: who performs them in the emergency department? Acad Emerg Med 1995;2:630-3.
8 Walls R, Vissers R, Sagarin M, et al. Emergency department intubations: final report of the national emergency airways registry pilot project. $\mathcal{F}$ Accid Emerg Med 1998;15:392 (abstract).

9 Sakles JC, Laurin EG, Rantapaa AA, et al. Airway management in the emergency department: a one year study of 610 tracheal intubation. Ann Emerg Med 1998;31: study of 610

10 Swartz DE, Matthay MA, Cohen NH. Death and other complications of emergency airway management in critically ill adults. Anaesthesiology 1995;82:367-76.

11 Gronlund NE. Stating behavioural objectives for classroom instruction. MacMillan, 1970.

12 Devitt JH, Kurrek MM, Cohen MM. Testing the raters: inter-rater reliability of standardised anaesthesia performance. Can $\mathcal{f}$ Anaesth 1997;44:924-8.

13 Devitt JH, Kurrek MM, Cohen MM. Testing internal consistency and construct validity during evaluation of performance in anesthetic patient simulation. Anesth Analg 1998;86:1160-4.

\title{
TOXBASE on the internet
}

\author{
A M Good, D N Bateman
}

TOXBASE, the clinical toxicology database of the UK National Poisons Information Service (NPIS), went on-line in 1983 using Viewdata technology. ${ }^{1}$ Since then it has provided a 24 hour service, access being available at the cost of a local telephone call to any registered medical professional within the NHS with a Viewdata terminal or personal computer (PC). ${ }^{2}$ There are currently more than 570 registered users who make around $50000 \mathrm{compu}$ ter accesses each year.

TOXBASE consists of approximately 13000 substance/product monographs on pharmaceuticals, agricultural and industrial chemicals, household products, toiletries, plants, and venomous animals. Each monograph contains information on toxic ingredients, toxicity, features after overdose, and clinical management. In addition there is general information on gut decontamination, slang terms for drugs, paediatric poisoning, nontoxic plants, poisoning in pregnancy, laboratory services for selected centres, and availability of antidotes for selected toxins.

The top 10 substance types that were the subject of TOXBASE inquiries during 1998 were (in decreasing order) paracetamol, codeine/dihydrocodeine, salicylates, ibuprofen, diazepam, zopiclone, petroleum distillates, caffeine (as a component of compound analgesics), fluoxetine, and dothiepin. The top three have held their positions since at least 1995 . Inquiries about dothiepin are decreasing and zopiclone increasing. ${ }^{3}$

The six centres of the NPIS also deal with more than 200000 telephone inquiries on poisonings every year, and this workload has been increasing. Many of these inquiries are straightforward, but the call load prevents rapid access for inquiries about more serious cases. To address this issue the UK Departments of Health decided to promote the use of TOXBASE to hospitals and general practitioners for answering poisons inquiries, particularly those involving less toxic substances. This will leave the more complicated issues involving serious poisonings and combinations of drugs for information staff and doctors.

TOXBASE is now available on the internet (http://www.spib.axl.co.uk/). To use the new TOXBASE a PC with Windows 95,98 , or NT and a frames enabled, Java compliant internet browser is required. This will typically be Microsoft Explorer version 4 (or above) or Netscape version 4 (or above). Access to the internet via either an NHSnet connection or a modem and access to an internet service provider is also needed. Those already registered for the old Viewdata version have been kept informed of developments and can use their current user name and password to access the database. Others, within the NHS, can register on-line. The existing Viewdata service will cease to operate at the end of 1999.

The new internet version of TOXBASE, with improved search and printing facilities, should lead to increased uptake, particularly in England and Wales (most in Scotland and Northern Ireland already use it). The ability to print key details on the management of a poisoning will assist in patient care, and promote best practice. It will remove the need for a "routine" call to the NPIS. NPIS centres will be involved in the promotion of TOXBASE in their locality. The new internet system will also have a feedback facility, which it is hoped will assist in data collection on the toxicology of unusual poisons and of new drugs. In this way the information provided on TOXBASE will be updated to reflect UK experience. Work will then start on a new improved version of the database, in consultation with UK toxicologists and TOXBASE user groups. Accident and emergency staff will therefore have a key role in developing the TOXBASE system for the future.

1 Proudfoot AT, Davidson WSM. A viewdata system for poisons information. BMF 1983;286:1125-7.

2 TOXBASE for poisons. Health Bulletin 1994;52:393.

3 Scottish Poisons Information Bureau. Annual report. Edinburgh: Scottish Poisons Information Bureau, 1998. 